\title{
Non-Neoplastic Muscle Disorder
}

National Cancer Institute

\section{Source}

National Cancer Institute. Non-Neoplastic Muscle Disorder. NCI Thesaurus. Code

C118317.

A non-neoplastic disorder that affects the smooth, skeletal, or cardiac muscles. 